\title{
-Original-
}

\section{Long-term Results of Ankle Arthrodesis Using an Intramedullary Nail with Fins in Patients with Rheumatoid Arthritis Hindfoot Deformity}

\author{
Kenji Takenouchi ${ }^{1,2}$, Minoru Morishita ${ }^{1,2}$, Kimihisa Saitoh ${ }^{3}$, \\ Kouichi Wauke ${ }^{3}$, Hiroshi Takahashi ${ }^{3}$ and Masakazu Nagashima ${ }^{1-3}$ \\ ${ }^{1}$ Department of Neurological, Nephrological and Rheumatological Science, Graduate School of Medicine, Nippon Medical School \\ ${ }^{2}$ Department of Joint Disease and Rheumatism, Nippon Medical School Hospital \\ ${ }^{3}$ Department of Rheumatology, Tokyo Metropolitan Bokutoh Hospital
}

\begin{abstract}
We report herein the results of a retrospective study of 30 ankle arthrodesis procedures performed in 27 patients with rheumatoid arthritis from 1994 through 2001 using a novel design of intramedullary nail with fins. The surgical treatment, post-operative management and clinical evaluation are described. The clinical evaluation, at an average follow-up period of more than 10 years, was based on foot disease scores from the Japanese Orthopaedic Association and scores obtained preoperatively, postoperatively, and during follow-ups 1 (November 2001) and 2 (November 2007) were compared. These variables showed significant improvement between before surgery and at follow-up evaluations. Non-union was not observed and no marked changes of the Chopart joint were seen between before surgery and at follow-up evaluations. Delayed wound healing was seen in 9 of 30 joints. However, infection neuropathy or other complications were not found. We conclude that arthrodesis using an intramedullary nail with fins is an effective treatment for severe hindfoot deformities in patients with rheumatoid arthritis because no cases of non-union were observed and because clinical results over the mean 10-year follow-up period were good or satisfactory.

(J Nippon Med Sch 2009; 76: 240-246)
\end{abstract}

Key words: rheumatoid arthritis, hind foot deformity, ankle arthrodesis, long-term results, intramedullary nail with fins

\section{Introduction}

Patients with rheumatoid arthritis (RA) usually have forefoot or hindfoot deformities. Forefoot deformities are usually treated with resection arthroplasty or telescoping osteotomy, whereas forefoot deformities are treated with total ankle arthroplasty (TAA) or ankle arthrodesis. TAA is considered to have limited indication, because the long-term outcome of TAA in most series has been poor, especially in young patients. The failure rate of TAA is high, with disappointing long term results, and the rate of complications, including loosening, sinking, and migration of the prosthesis, is also high $^{2,3}$.

Correspondence to Masakazu Nagashima, MD, Department of Rheumatology, Tokyo Metropolitan Bokutoh Hospital, 4-23-15 Kotohbashi Sumida-ku, Tokyo 130-8576, Japan

E-mail: m_nagashima@bokutoh-hp.metro.tokyo.jp

Journal Website (http://www.nms.ac.jp/jnms/) 
Table 1 The indication for ankle arthrodesis and total ankle arthroplasty on patients with rheumatoid arthritis

\begin{tabular}{ll}
\multicolumn{1}{c}{ Ankle arthrodesis } & \multicolumn{1}{c}{ Total ankle arthroplasty } \\
\hline Severe varus or valgus deformity & Non-severe varus or valgus deformity \\
Destructive talocalcaneal joint & Possible fusion of talocalcaneal joint \\
Instability or mutilans deformity & Possible within 15 degrees of varus or valgus deformity \\
Severe osteoporosis or osteonecrosis of the talus & Non-severe osteoporosis or non osteonecrosis of the talus \\
Developmental RA activity & Lower RA activity \\
Non-limited indicative age & Indicative age around 60 years \\
Failed total ankle arthroplasty & Hemilateral ankle arthrodesis \\
\hline
\end{tabular}

On the other hand, ankle arthrodesis has been performed to relieve pain and cure severe deformities of the hindfoot without such limited indications ${ }^{4-7}$. However, such methods as compression arthrodesis and transfibular or anteromedial sliding graft arthrodesis have disadvantages that include the need for a long period of immobilization without weight-bearing and a high incidence of nonunion $(10 \%-20 \%)^{8}$. To overcome these problems, tibiotalocalcaneal arthrodesis with a retrograde intramedullary nail has been developed ${ }^{1,9-14}$. Additionally, an intramedullary nail with fins has been developed. The most significant characteristic of this cylindrical nail is that it has 4 fins with sharp tips. These fins prevents motion of the ankle joint in a range of directions. The transfixation screws of this nail may help control rotation, pronation supination, dorsiflexion, and plantar flexion, but their major role is to prevent the nail from sliding distally ${ }^{1}$.

We report herein the results of 30 cases of ankle arthrodesis for severe deformities of the ankle joint in patients with RA using an intramedullary nail with fins over a follow-up period of approximately 10 years.

\section{Patients and Methods}

From May 1994 through June 2001, 27 patients (26 women and 1 man) with RA underwent ankle arthrodesis using an intramedullary nail with fins at Nippon Medical School Hospital. Two patients underwent bilateral ankle arthrodesis, and 1 patient underwent ankle arthrodesis after resection of an infected TAA, for a total of 30 joints. The average age at surgical treatment was $59.8 \pm 9.4$ years (range, 43-76 years), the average follow-up period was $10.7 \pm 2.4$ years (range, 6.5-13.8 years), and the average operation time was $3.0 \pm 0.8 \mathrm{hrs}$ (range, 1.75.1 hours). The severity of radiographic changes of the talocrural joint according to the Larsen classification $^{15}$ was grade 3 was 8 joints, grade 4 in 20 joints, and grade 5 in 2 joints. Changes at the talocalcaneal joint were grade 1 in 0 joints, grade 2 , in 2 joints, grade 3 , in 13 joints, grade 4 , in 13 joints, and grade 5 , in 2 joints. Five patients had died and 6 joints were excluded from the study by the end of the follow up period. Severe hind foot deformity was indicated by Larsen grade greater than 4 , a varus or valgus deformity greater than 15 degrees.

The indications for surgery in all patients are shown in Table 1. The indications included severe varus or valgus deformity of the talocrural or talocalcaneal joint; instability or mutilans deformity; severe osteoporosis or osteonecrosis of the talus; further development of RA; and failed TAA.

\section{Characteristics of the Intramedullary Nail with}

\section{Fins and the Surgical Technique}

The distal part of the nail has four $6 \mathrm{~cm}$ long fins attached. These fins prevent rotation between the tibia and talus bones and stabilize the talocrural joint. These actions results in easier compression between the tibia and talus bones, earlier weightbearing, and easier bone fusion than with other ankle arthrodesis techniques.

For surgery, a longitudinal anterior approach was used through the skin, subcutaneous tissues and the ankle joint, which was entered lateral to the anterior neurovascular bundle. The distal end of the tibia was cut perpendicular to its long axis, and this cut was sometimes extended to part of the malleolus, with only enough bone removed to create a flat 
surface. The talar dome was cut parallel to the tibia cut and the planta pedis, again with minimal bone removed to create a flat surface. The fibula was sometimes cut at the same level as or at a level higher than the tibia so that the cut surfaces of the tibia and talus were at better positions. If the distal fibula was removed, it was used as a bone graft in some cases. The optimal position of the foot was a neutral position of the ankle with a neutral or 2 to 3 degree valgus hindfoot. Staples were sometimes used to fix the talocrural or talocalcaneal joint and, thus. to maintain the foot in the correct position.

After correction of the talocrural and talocalcaneal joints, internal fixation was performed as follows. A guide wire was retrogradely inserted from the calcaneus to the tibia via the talus under an image intensifier. After reaming, a nail of the appropriate length and width was inserted over the guide wire and passed across the tibiotalocalcaneal joint to the tibial medullary canal using an impactor for the nail. The distal end of the nail was driven $5 \mathrm{~mm}$ into the calcaneus to prevent the nail from sliding distally. Transfixation screws to hold the proximal area of the nail and an end cap were inserted by means of a targeting device.

\section{Postoperative Treatment}

Patients were asked to bear weight as toleated, whether or not they had received bone grafts. In this study, 18 of 30 joints were able to bear weight 3 weeks after surgery, and 12 joints with grafted bone were able to bear weight after 6 or 8 weeks. If there was concern that solid internal fixation could not be obtained because of poor bone stock, a below-theknee cast was applied for 6 to 12 weeks postoperatively until fusion was confirmed with radiography, after which weight-bearing was started. Osseous fusion was considered to be present when trabeculae crossing the site of arthrodesis were seen radiographically, resulting in the disappearance of the gap. Two surgical rheumatologists independently evaluated the radiographs.

\section{Clinical Assessment and Statistical Analysis}

Clinical assessment was performed using the 100- point rating system for foot disease of the Japanese Orthopaedic Association (JOA): pain (0-20 points); deformity (forefoot, 0-10 points; hindfoot, 0-20 points); range of motion (forefoot, 0-5 points; hindfoot, $0-5$ points); instability (0-10 points); walking ability (0-10 points); muscle strength (0-5 points); sensation (0-5 points); and activity of daily living (010 points). With this scoring system, the higher was the score, the greater was the improvement in symptoms.

The Wilcoxon signed rank test was used to compare the total scores and the score of each variable at before surgery, after surgery (at the time of discharge after surgery), and at follow-up periods 1 (November 2001) and 2 (November 2007). $P$ values of less than 0.05 were considered to indicate a significant difference.

\section{Results}

The peri-operative management and assessment of arthrodesis were as follows: 12 of 30 joints were treated with bone grafts using chips from a part of the distal end of the fibula and 8 were treated with internal fixation using staples and a nail. Five of 8 joints were immobilized with a below-the-knee cast for 6 to 12 weeks. Weight-bearing was started an average of 3.5 weeks after the operation, with a range of 1 to 12 weeks. Arthrodesis of the ankle in an optimal position, namely a neutral position or a position 2 to 3 degrees valgus hindfoot to the tibia, was achieved in all patients, and all patients showed osseous fusion within 14 weeks postoperatively.

Twenty-eight of 30 joints in which talocrural arthrodeses were performed also exhibited fusion of the talocalcaneal joints. However, in 2 cases of mutilans deformity the talocalcaneal joint did not exhibit osseous fusion, but no symptoms caused by the talocalcaneal joints were observed. In 5 of the 30 joints, spontaneous arthrodesis of the adjacent talonavicular joint was recognized after tibiotalocalcaneal joint arthrodesis was performed. The talonavicular joint in the remaining 25 cases was not markedly changed between before surgery and after surgery. Before surgery, osteoarthritic change of the Chopart joint was recognized in 19 of 

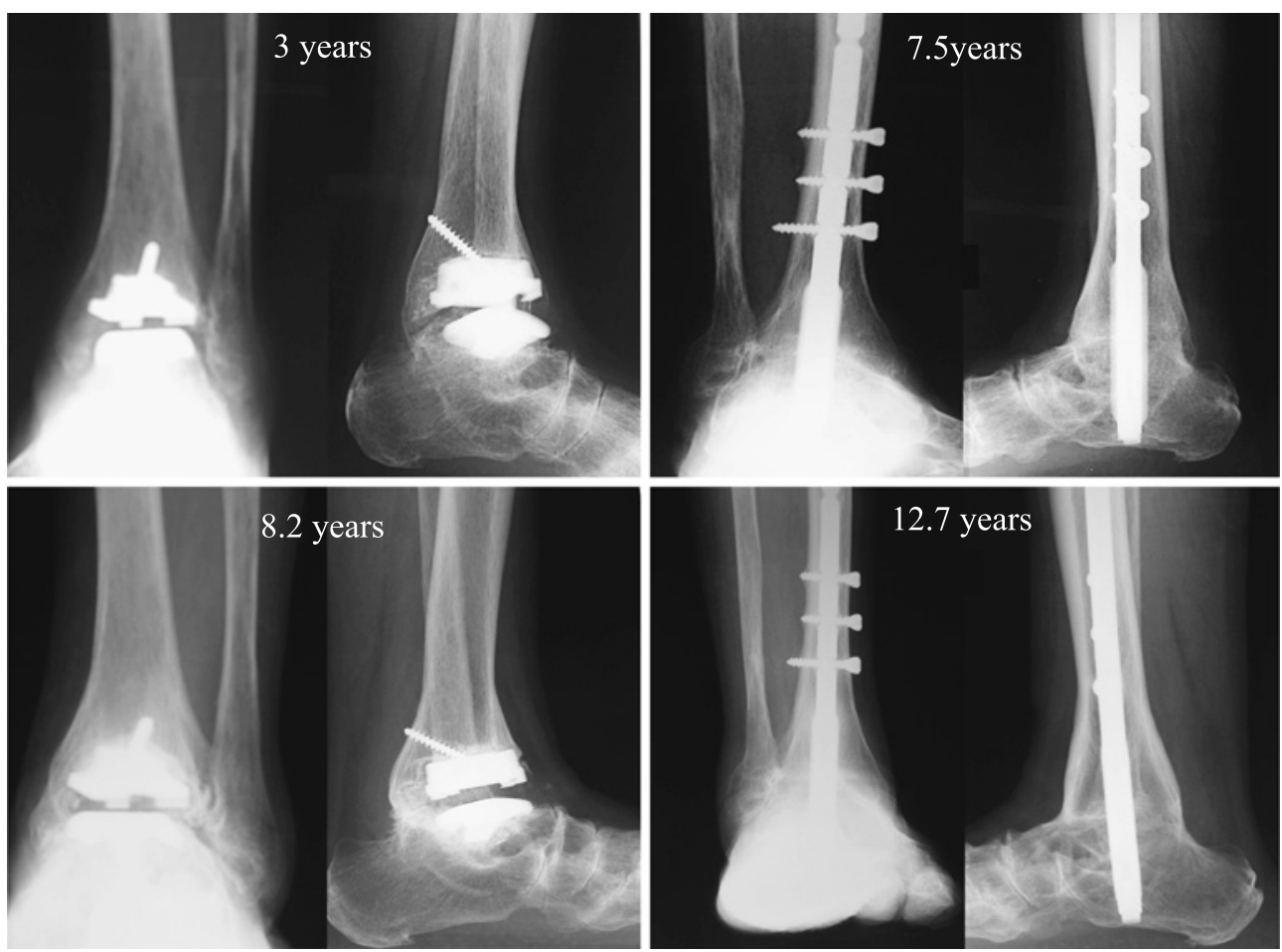

Fig. 1 Case 1. Postoperative radiographs of ankle arthrodesis and TAA in a 51-year-old woman with RA.

The bilateral talocrural and talocalcaneal joints show fibrous ankylosis, with the right ankle joint showing especially destructive changes. The patient was relatively young and had a high level of activities of her daily living. This patient was considered a good candidate for ankle arthrodesis and TAA. Ankle arthrodesis was performed on the right ankle when the patient was aged 51 years, and radiographs obtained 7.5 and 12.7 years after surgery are shown. TAA was performed on the left ankle when the patient was aged 56 years, namely 5 years after ankle arthrodesis, and radiographs obtained 3 and 8.2 years after surgery are shown.

these 25 joints, and spontaneous fusion of the Chopart joint were recognized in 6 joints. In the figures we show 2 cases of this surgery. The first case shows TAA and ankle arthrodesis-performed in the same patients (Fig. 1). The second case shows radiographs obtain before surgery and 7 years after surgery (Fig. 2). Both of these cases show panarthrodesis of the talocrural and talocalcaneal joints. -Figure 1, 2 show radiographs obtained before surgery and at follow-up. All of these cases show panarthrodesis of the talocrural and subtalar joints.

In the clinical assessment (Fig. 3), total JOA scores were significantly improved in all patients from $35.4 \pm 10.4$ points before surgery to $64.3 \pm 9.30$ points after surgery, and then to $59.3 \pm 8.30$ points and $55.2 \pm 10.1$ points during the follow-up period. Pain scores also significantly improved from $5.67 \pm$
3.14 points before surgery to $17.8 \pm 2.84$ points after surgery, and $17.3 \pm 3.43$ points and $18.1 \pm 3.25$ points in the follow-up period. The mean score of the hindfoot deformity also increased from $6.23 \pm 5.43$ points to $17.1 \pm 3.03$, to $16.7 \pm 3.2$, and to $14.3 \pm 3.73$, respectively. Walking ability increased from $2.70 \pm$ 2.02 points to $5.0 \pm 1.72,4.20 \pm 1.82$, and $4.31 \pm 1.80$, respectively. Walking ability and activities of daily living significantly improved when scores from before surgery and after surgery were compared and when scores from before surgery and follow-up periods 1 and 2 were compared.

Instability scores improved from $3.93 \pm 3.13$ points before surgery to $6.33 \pm 3.20$ points after surgery and to $4.60 \pm 2.90$ points and $6.30 \pm 3.15$ during the follow-up period (Fig. 3). However, a forefoot deformity developed during follow-up period 2 . Muscle strength and sensation remained unchanged 

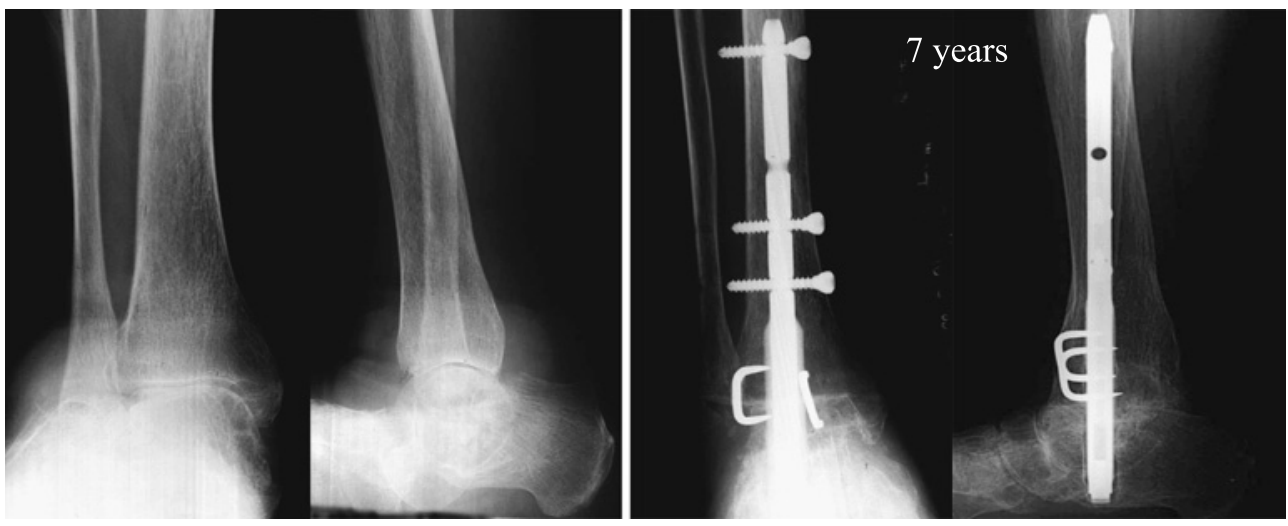

Fig. 2 Case 2. A 68-year-old woman with RA. Evaluation of preoperative radiographs shows severe valgus deformity, and the patient reported severe pain, considered to be an which is an indication for ankle arthrodesis. A bone graft and augmentation with staples were performed between the tibia and talus bones. Radiographs from before surgery and 7 years after surgery are shown.

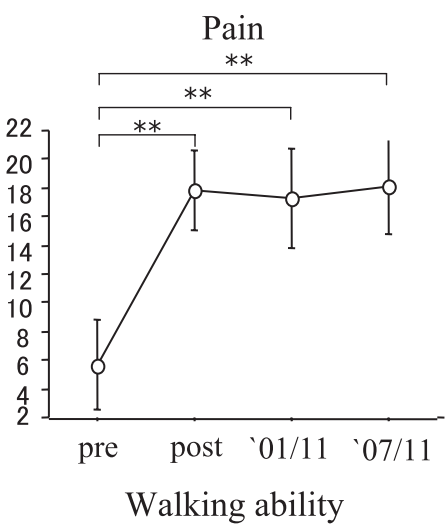

Forefoot deformity

Hindfoot deformity
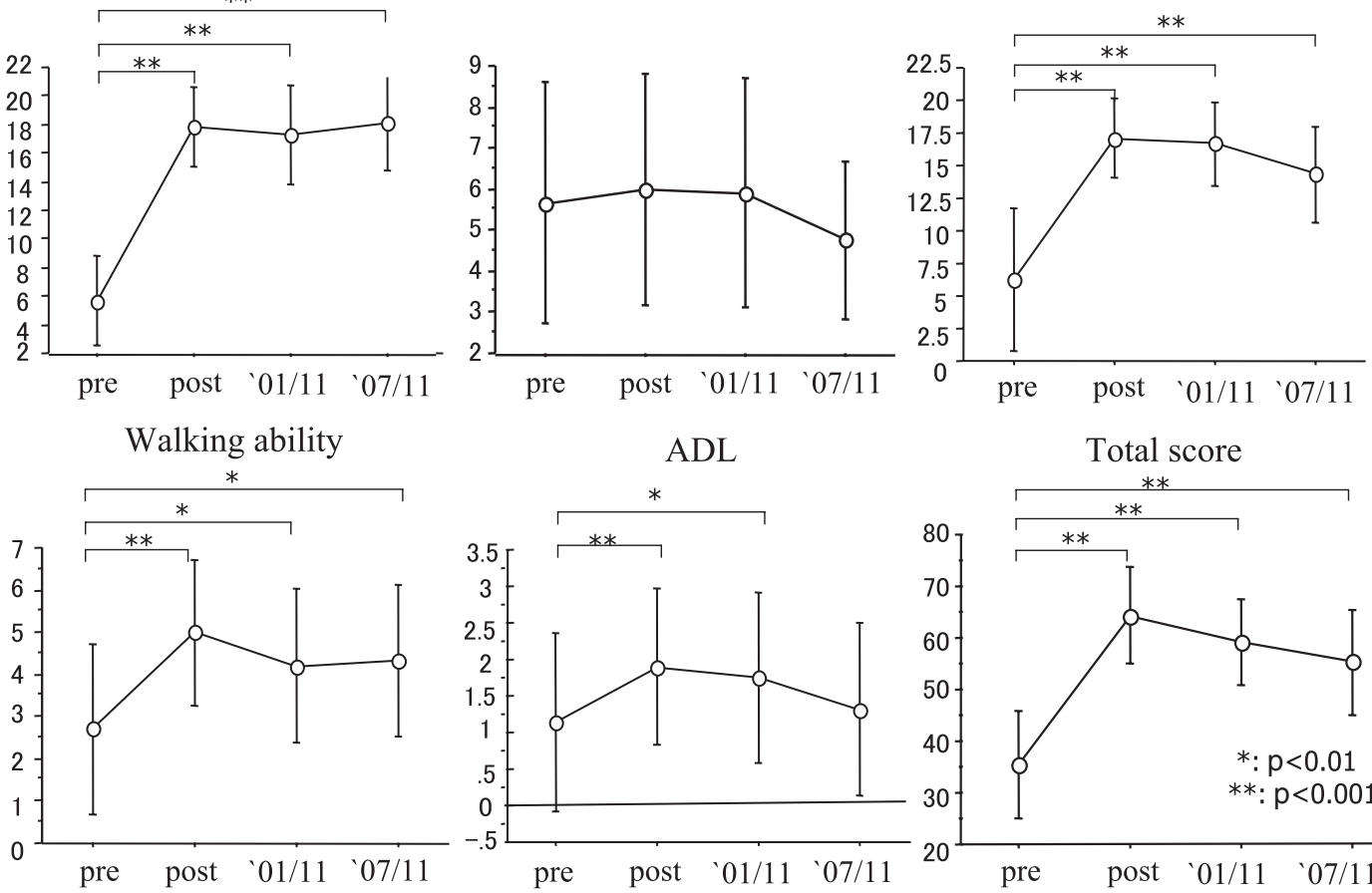

ADL
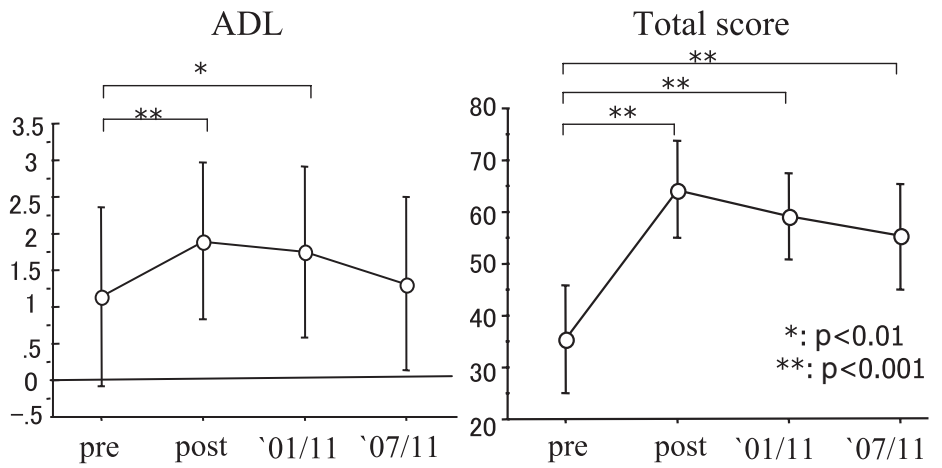

Fig. 3 Changes in clinical scores based on the JOA scoring system before surgery, after surgery, and during the follow-up periods (November 2001 and November 2007). Total score, pain score, hindfoot deformity, walking ability, and activity of daily living score were all significantly improved when score from before surgery and score from the follow-up periods were compared. However, forefoot deformity developed during the follow-up periods after surgery.

in the majority of patients between before and after surgery and between before surgery and during follow-up. All patients were satisfied with the outcome of the operation. Nine patients had delayed wound healing that resolved with dressing changes. No other complications occurred.

\section{Discussion}

In patients with RA who have severe pain caused by a forefoot or hindfoot deformity resulting in restricted walking ability without knee pain, relief 
from is of primary concern. Ankle arthrodesis, in particular, that with an intramedullary nail with fins, has several advantages for patients with RA: a shortening of the period of immobilization, earlier weight-bearing, and exact bony fusion of the ankle joint. The operating time with this nail is shorter than that with other ankle arthrodesis, because this nail has no screw of the distal area. In recent years, the rates of fusion of other ankle arthrodesis methods have been reported to be $90 \%$ to $95 \%{ }^{16-19}$, with long periods of no weight-bearing and immobilization required after surgery.

In our procedures, we attempted to achieve firm internal fixation, earlier weight-bearing, and a prevention of pronation and supination of the ankle joint using a nail with 4 distal fins. However, we carefully considered whether this intramedullary nail should be used for patient with RA and severe osteoporosis induced by disuse bone atrophy or steroid treatment. Because of the poor fixation in osteoporotic bone and the possibility of necrosis of the overlying skin caused by a proximal screw head just beneath the skin, we conclude that transverse screws should not be used in these cases.

Several authors have reported ankle arthrodesis using an intramedullary nail ${ }^{11-13}$. One disadvantage of retrograde intramedullary nailing for ankle arthrodesis is the necessity to fuse the subtalar joint along with the ankle joint. However, compression arthrodesis of the ankle joint limits subtalar movement ${ }^{20}$. Rigid talocrural arthrodesis may induce development of a varus or valgus deformity of the subtalar joint in patients with RA. Horibe et al. have reported that destruction of the adjacent joints, namely the Chopart, subtalar, and Lisfranc joints, is accelerated by talocrural arthrodesis ${ }^{21}$. Adam et al. have reported that subluxation of the subtalar joint occurs in about $80 \%$ of cases after talocrural arthrodesis, resulting in a varus or valgus deformity of the subtalar joint ${ }^{22}$, and Felix et al. have reported that while 2 of 14 patients had normal subtalar joints, 8 patients showed spontaneous fusions following talocrural arthrodesis ${ }^{23}$. On the basis of these results, it may be reasonable to fix the talocrural and subtalar joints at the same time. Most of the subtalar joints in our patients with RA had
Larsen grades 3 higher than 3, and destruction and deformity of the subtalar joints were considered to be progressing; therefore, rigid fixation of only the talocrural joint may accelerate deformation of the subtalar joint. Shibata has reported that Chopart joints that underwent solid fusion exhibited no marked changes at an average follow-up period of more than 9 years ${ }^{1}$.

Comparing the results of a mean follow-up period of 10.7 years to those of a mean follow-up of 5.1 years, the pain scores, hindfoot deformity, walking ability, and activities of daily living scores were significantly improved and this improvement was maintained for more than 10 years after surgery. Muscle strength and sensory disturbances had not markedly changed between these time points, whereas forefoot deformities progressed over the long-term.

One of the most severe complications that can occur is non-union or delayed union. Superficial infection, neuropathy, and delayed wound healing have been reported after ankle arthrodesis for RA. Moran et al. followed up 30 patients with RA after ankle arthrodesis for 5 years and found 6 cases of non-union, 6 of fibrous union, 12 of wound breakdown and infection ${ }^{24}$. Cracchiolo et al. have reported that in 32 cases of arthrodesis of the ankle joint in 26 patients with RA, the rate of non-union was $22 \%$, that of deep and superficial infection was $29 \%$, that of malalignment was $16 \%$, and that of neurapraxia was $9 \%$. Felix et al. have reported delayed wound healing in 2 of 26 ankle joints, superficial ulceration in 1 joint, and sural neuritis in 1 joint $^{23}$. In our series, delayed wound healing was recognized in 9 of 30 joints. However, infection, neuropathy, or other complications did not occur. Ankle arthrodesis also does not provide ideal results with regard to the anatomy and function of the ankle joint. Despite these limitations, this method is effective for relieving pain in patients with severe hindfoot deformities.

We conclude that tibiotalocalcaneal arthrodesis using an intramedullary nail with fins is a good treatment for severe hindfoot deformities in patients with RA, because non-union was not observed and the clinical results over an average 10-year follow-up 
period were good or satisfactory, other than the continuing development of forefoot deformities.

\section{References}

1. Fujimori J, Yoshino S, Koiwa M, Nakamura H, Shiga $\mathrm{H}$, Nagashima M: Ankle arthrodeis in rheumatoid arthritis using an intramedullary nail with fins. Foot Ankle Int 1999; 20: 485-490.

2. Hamblane DL: Can the ankle joint be replaced? J Bone Joint Surg Br 1985; 67: 689-690.

3. Jensen NC, Kroner K: Total ankle joint replacement: a clinical follow-up. Orthopedics 1992; 15: 236-239.

4. Charnley J: Compression arthrodesis of the ankle and shoulder. J Bone Joint Surg Br 1951; 33: 180-191.

5. Adams JC: Arthrodesis of the ankle joints. Experience with the transfibular approach. J Bone Joint Surg Br 1948; 30: 506-511.

6. Kennedy JC: Arthrodesis of the ankle with particular reference to the Gallie procedure. J Bone Joint Surg Am 1990; 42: 1308-1316.

7. Patterson RL Jr, Parrish FF, Hathaway EN: Stabilizing operation on the foot: A study of the indications, techniques used, and end results. Foot Ankle Int 1996; 17: 594-607.

8. Lance EM, Paval A, Fries I, Larsen I, Patterson RL Jr: Arthrodesis of the ankle joint; A follow up study. Clin Orthop 1979; 142: 146-158.

9. Mendicino RW, Catanzariti AR, Saltrick KR, et al: Tibiotalocalcaneal arthrodesis with retrograde intramedullary nailing. J Foot Ankle Surg 2004; 43: $82-86$.

10. Nagashima M, Tachihara A, Matsuzaki T, et al.: Follow-up study of ankle arthrodesis in severe hind foot deformity in patients with rheumatoid arthritis using an intramedullary nail with fins. Mod Rheumatol 2005; 15: 269-274.

11. Shibata T, Tada K, Hashizume C: The results of arthrodesis of the ankle for leprotic neuroarthroplasty. J Bone joint Surg Am 1990; 72: 749-756.

12. Moore TJ, Prince R, Pochatko D, Smith JW, Fleming
S: Retrograde intramedullary nailing for ankle arthrodesis. Foot Ankle Int 1995; 16: 433-436.

13. Stone K, Helal B: A method of ankle stabilization. Clin Orthop 1991; 268: 102-106.

14. Chou LB, Mann RA, Yaszay B, et al:: Tibiotalocalcaneal arthrodesis. Foot Ankl Int 2000; 21: 804808.

15. Larsen A, Dale K, Eek M: Radiographic evaluation of rheumatoid arthritis and related conditions by standard reference films. Acta Radiol Diagn 1977; 18: 481-491.

16. Dennis DA, Clayton ML, Wong DA, Mack RP, Susman $\mathrm{MH}$ : Internal fixation compression arthrodesis of the ankle. Clin Orthop 1990; 253: 212220.

17. Holt ES, Hansen ST, Mayo KA, Sangeorzan BJ: Ankle arthrodesis using internal screw fixation. Clin Orthop 1991; 268: 21-28.

18. Moeckel BH, Patterson BM, Inglis AE, Sculco TP: Ankle arthrodesis. Clin Orthop 1991; 268: 78-83.

19. Quill GE: Tibiotalocalcaneal and pantalar arthrodesis. Foot Ankl Clin 1996; 1: 199-209.

20. Ratliff AHC: Compression arthrodesis of the ankle. J Bone Joint Surg Br 1959; 41: 524-534.

21. Horibe S, Tada K, Nagano J: Neuroarthroplasty of the foot in lepropsy. J Bone Joint Surg Br 1988; 70: 481-485.

22. Adam W, Ranawat C: Arthrodesis of the hindfoot in rheumatoid arthritis. Orthop Clin North Am 1976; 7: 827-840.

23. Felix NK, Kitaoka HB: Ankle arthrodesis in patients with rheumatoid arthritis. Clin Orthop 1998; 349: 5864.

24. Moran CG, Pinder IM, Smith SR: Ankle arthrodesis in rheumatoid arthritis. 30 cases followed for 5 years. Acta Orthop Scand 1991; 62: 538-543.

25. Cracchiolo A III, Cimino WR, Lian G: Arthrodesis of the ankle in patients who have rheumatoid arthritis. J Bone Joint Surg Am 1992; 74: 903-909.

(Received, February 23, 2009)

(Accepted, June 16, 2009) 